\title{
THE JOURNAL
}

OF

\section{HELLENIC STUDIES}




\section{THE JOURNAL}

or

\section{HELLENIC \\ STUDIES}

VOLUME XX. (1900)

PUBLISHED BY THE COUNCIL, AND SOLD ON THEIR BEHALF

BY

MACMILLAN AND CO., Limited, ST. MARTIN'S STREET LONDON, W.C.

MDCccC

The Rights of Translation and Reproduction are Reserved 
Riohard Clay and Sons, Limited, LONDON AND BUNGAY. 


\section{CONTENTS.}

Rules of the Society ... ...

$\begin{array}{lllllllllll}\text { List of Officers and Members } & \ldots & \ldots & \ldots & \ldots & \ldots & \ldots & \ldots & \ldots & \ldots & \mathrm{xv}\end{array}$

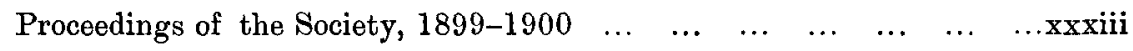

Proceedings of the Cambridge Branch of the Society $\ldots \begin{array}{lllll}\ldots & \ldots & \ldots & \ldots & \mathrm{xliv}\end{array}$

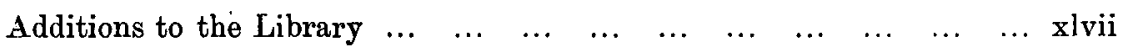

Collection of Photographs and Lantern-Slides...

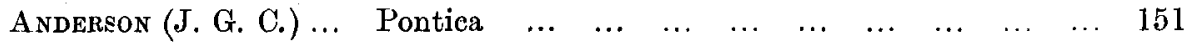

$\begin{array}{llllllllll}\text { Awdry (H.) } & \ldots & \text { Pylos and Sphacteria } & \ldots & \ldots & \ldots & \ldots & \ldots & \ldots & 14\end{array}$

$\begin{array}{lllllllll}\text { Bevan (E. R.) } & \ldots & \text { A Note on Antiochos Epiphanes } & \ldots & \ldots & \ldots & \ldots & 26\end{array}$

Bosanquet (R. C.) $\quad \ldots \quad$ Archaeology in Greece, 1899-1900 $\quad \ldots \quad \ldots \quad \ldots \quad 167$

$\begin{array}{lllllllllllll}\operatorname{CooK}(\text { A. В.) } & \ldots & \text { Iostephanos } & \ldots & \ldots & \ldots & \ldots & \ldots & \ldots & \ldots & \ldots & & 1\end{array}$

Crowfoot (J. W.) _.. Some Portraits of the Flavian Age (Plates I-IV.) 31

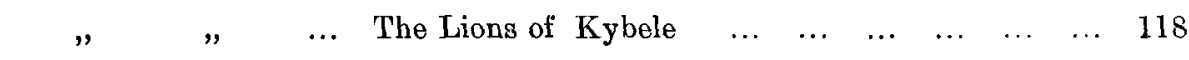

$\begin{array}{llllllll}\text { Edmonds (C. D.) } & \ldots & \text { The Tumulus of Piláf-Tepé (Plate V.) } & \ldots & \ldots & \ldots & 20\end{array}$

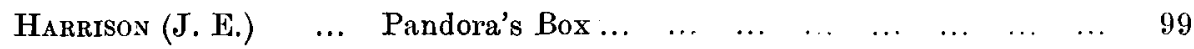

$\begin{array}{lllllllll}\text { Munro (J. A. R.) } \quad \ldots & \text { Some Pontic Milestones ... } & \ldots & \ldots & \ldots & \ldots & \ldots & 159\end{array}$

Mrres (J. L.)... $\quad \ldots \quad$ On the Plan of the Homeric House $\quad \ldots \quad \ldots \quad \ldots \quad 128$

$\begin{array}{lllllll}\text { Paton (W. R.) } \quad \ldots & \text { Sites in E. Karia and S. Lydia (Plate VI.) ... } & \ldots & 57\end{array}$

$\begin{array}{llllll}\text { Robert (C.) } & \ldots & \ldots & \text { Roman Sarcophagi at Clieveden (Plates VII-XII.) } & 81\end{array}$

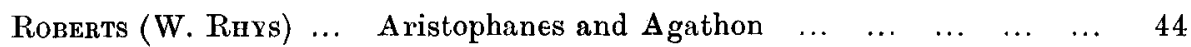

$\begin{array}{llllllllll}\text { Verrall (A. W.) } & \ldots & \text { The Name Anthesteria } & \ldots & \ldots & \ldots & \ldots & \ldots & \ldots & 115\end{array}$

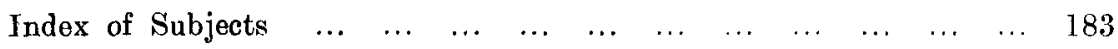

$\begin{array}{llllllllllllll}\text { Greek Index } & \ldots & \ldots & \ldots & \ldots & \ldots & \ldots & \ldots & \ldots & \ldots & \ldots & \ldots & \ldots & 187\end{array}$ 


\section{LIST OF PLATES.}

I., II. Portrait Heads in the Uffizi.

III. Portrait Head in the Jacobsen Collection.

IV. The 'Mark Antony' in the Vatican.

V. Silver Vase from Piláf-Tepé.

VI. Map of E. Karia and S. Lydia.

VII-XII. Sarcophagi at Clieveden. 


\section{LIST OF ILLUSTRATIONS IN THE TEXT.}

Sketch Map of Pylos and Sphacteria

$\begin{array}{llllllllll}\text { Tumulus of Piláf-Tepé from the South } & \ldots & \ldots & \ldots & \ldots & . . & \ldots & \ldots & \ldots & 20\end{array}$

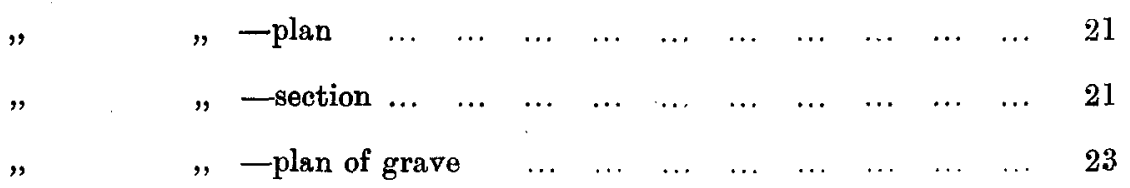

Perforated Vase from Pilá-Tepé

$\begin{array}{lllllllllll}\text { Route-map from Euren to Aidin } & \ldots & \ldots & \ldots & \ldots & \ldots & \ldots & \ldots & \ldots & \ldots & 58\end{array}$

$\begin{array}{llllllllllllll}\text { Plan of Koskinia } & \ldots & \ldots & \ldots & \ldots & \ldots & \ldots & \ldots & \ldots & \ldots & \ldots & \ldots & \ldots & 59\end{array}$

Route-map from Anineta to Üzüm Ova

$\begin{array}{llllllllllll}\text { Tumulus of Altyn-Tash-view } & \ldots & \ldots & \ldots & \ldots & \ldots & \ldots & \ldots & \ldots & \ldots & \ldots & 66\end{array}$

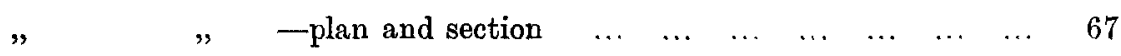

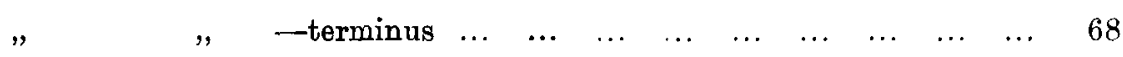

$\begin{array}{lllllllllllll}\text { Tumulus at Kavakly-plan } & \ldots & \ldots & \ldots & \ldots & \ldots & \ldots & \ldots & \ldots & \ldots & \ldots & 68\end{array}$

$\begin{array}{llllllllllll}\text { Tumulus at Arably }- \text { plan } \ldots & \ldots & \ldots & \ldots & \ldots & \ldots & \ldots & \ldots & \ldots & \ldots & \ldots & 69\end{array}$

Terminus (Von Olfert, Abh. pr. Akad. 1858)

$\begin{array}{lllllllllll}\text { Terminus of Alabanda Tumulus } & \ldots & \ldots & \ldots & \ldots & \ldots & \ldots & \ldots & \ldots & \ldots & 70\end{array}$

$\begin{array}{lllllllllllll}\text { Tomb at Yelije-plan } & \ldots & \ldots & \ldots & \ldots & \ldots & \ldots & \ldots & \ldots & \ldots & \ldots & \ldots & 70\end{array}$

$\begin{array}{llllllllllll}\text { Tomb at Asma-Kale-plan } & \ldots & \ldots & \ldots & \ldots & \ldots & \ldots & \ldots & \ldots & \ldots & \ldots & 71\end{array}$

$\begin{array}{llllllllll}\text { Tomb at Bin-ghetch-plan and section } & \ldots & \ldots & \ldots & \ldots & \ldots & \ldots & \ldots & \ldots & 71\end{array}$

$\begin{array}{llllllllll}\text { Tomb at Sarij-ova-plan and section } & \ldots & \ldots & \ldots & \ldots & \ldots & \ldots & \ldots & \ldots & 72\end{array}$

$\begin{array}{lllllllllllll}\text { Sarcophagus at Bin-ghetch } & \ldots & \ldots & \ldots & \ldots & \ldots & \ldots & \ldots & \ldots & \ldots & \ldots & 76\end{array}$

$\begin{array}{lllll}\text { Part of the Theseus Sarcophagus at Clieveden before restoration... } & \ldots & \ldots & 90\end{array}$

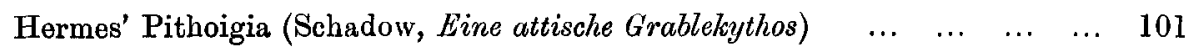

$\begin{array}{llllllllllll}\text { Pandora and the Hammerers } & \ldots & \ldots & \ldots & \ldots & \ldots & \ldots & \ldots & \ldots & \ldots & \ldots & 106\end{array}$ 
viii

CON'TENTS.

$\begin{array}{llllllllll}\text { Plan of the Homeric House }(\text { Gardner) } & \ldots & \ldots & \ldots & \ldots & \ldots & \ldots & \ldots & \ldots & 130\end{array}$

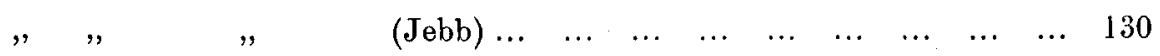

The Palace at Tiryns (J.H.S. vii. 172)

$\begin{array}{lllllllllllll}\text { The Palace at Mykenai } & \ldots & \ldots & \ldots & \ldots & \ldots & \ldots & \ldots & \ldots & \ldots & \ldots & \ldots & 131\end{array}$

The Megaron of the Palace at Tiryns (J.H.S. vii. 164) $\ldots \begin{array}{llllll}\ldots & \ldots & \ldots & \ldots & \ldots & 145\end{array}$

$\begin{array}{llllllllll} & , & & & & & & & & \end{array}$ 